\title{
Saccade latency and warning signals: Stimulus onset, offset, and change as warning events
}

\author{
LEONARD E. ROSS and SUSAN M. ROSS \\ University of Wisconsin, Madison, Wisconsin 53706
}

\begin{abstract}
Two experiments investigated saccade latency to a peripheral target under various warning signal conditions. In Experiment 1, the effects of warning stimulus onset, change, and two offset conditions were compared at warning intervals of $0,100,300$, and $600 \mathrm{msec}$. Warning stimulus onset, change, and offset were all effective in reducing saccade latency as compared to a no-warning control condition, but warning stimulus offset resulted in shorter saccade latency than onset or change at all warning intervals. Experiment 2 compared onset and offset warning conditions at $-300-,-250-,-200-,-150-,-100-,-50-, 0-$, and 50-msec intervals. Responses following onset were slower than those following offset at the latter five intervals, while warning onset resulted in slower saccades than no-warning control conditions at -150 -, $-100-$, and $-50-\mathrm{msec}$ intervals. These results indicate that the onset of a visual warning signal can have an interfering effect on the programming or execution of a saccade.
\end{abstract}

The latency of a saccade to a peripheral target is shorter when a warning signal precedes the onset of the target (Becker, 1972; Cohen \& Ross, 1977, 1978; Saslow, 1967). A corresponding warning-signal effect is commonly found in conventional manual reaction-time situations, at least under conditions where the warning signal does not disrupt a preparatory state of the subject that may have resulted from other foreperiod events (Sanders, 1972). However, in contrast to the numerous studies concerned with the effects of warning on manual reaction time, there has been little systematic investigation of warningsignal effects on the latency of the oculomotor response.

While it is possible that saccadic and manual responses have identical properties and display similar relationships to parameters of the warning stimulus, the response systems differ in ways that suggest the usefulness of further study of the saccadic response under various warning conditions. First, the saccadic response is generally considered more automatic than manual responses because of the ballistic-like characteristics of the saccade, its high degree of practice, and its independence of attentional-cognitive involvement under nonlaboratory conditions. Secondly, the speed-accuracy tradeoff and response criterion factors associated with manual reaction-time responses may not enter into saccadic responses under some circumstances due to the programmed characteristics of saccades. Relevant to this possibility is the finding of independence between saccadic response latency and accuracy under warning signal conditions (Cohen

This research was supported by USPHS Grant HD08240. Requests for reprints may be addressed to either author, Psychology Department, University of Wisconsin-Madison, Madison, Wisconsin 53706.
\& Ross, 1978). Finally, it should be noted that since the saccade is a functional response that places visual targets on the fovea for improved reception it might be affected by visual warning stimuli in a manner different from that of manual reaction-time responses.

In fact, the shorter latency of saccades following warning has been attributed to factors other than the alterting process commonly cited as the facilitating factor in other reaction-time situations. Saslow (1967) found that warning signal offset reduced saccade latency not only when the offset preceded target onset but even when it occurred $100 \mathrm{msec}$ after target onset. This finding was considered to be inconsistent with an alerting interpretation, and it was postulated that the warning effect was due to a reduction in the frequency of the small corrective saccades that occur during fixation. Fixation point offset, the warning event, could result in a reduction in the frequency of fixation saccades, and therefore correspondingly reduce their refractory effects on the saccade system. The latency of the saccade to the target stimulus would thus be reduced. The refractoriness accompanying fixation microsaccades was also discussed by Carpenter (1977) as a factor that might affect saccade latency following a warning event. The extent to which such an effect occurs under stimulus offset warning conditions is unknown, although it should be noted that all of the studies investigating the effects of warning on saccade latency have used fixation offset as the warning event, and it is possible that the reduced saccadic latency found following warning could reflect such a factor.

A refractory effect such as that discussed above should result in shorter saccade latencies following warning stimulus offset as compared to warning stimulus onset, due either to a reduction in microsaccades following stimulus offset or to an increase 
in microsaccades following the onset of a new stimulus. Other factors might be expected to favor stimulus offset over onset as a warning event. Studies employing temporal order discrimination to investigate the perceptual latencies of visual signals have found stimulus offset to be perceived earlier than onset (Hansteen, 1971; Lewis, Dunlap, \& Matteson, 1972; Walsh, 1973), and warning stimulus offset thus could be registered more quickly with a corresponding faster saccadic response. It is also possible that the onset of a stimulus could elicit a fixation response for a short period due to other central or peripheral processes that have the function of permitting the extraction of information from the new stimulus. In this respect, Potter and Levy (1969) noted that eye movements were spontaneously almost completely suppressed during the rapid presentation of pictures (4-8 pictures/sec). Assuming that the onset of a stimulus is more likely to presage new information, such a fixation effect might occur more readily to the onset or change of a stimulus than to the termination of a stimulus already fixated for some period.

In view of the lack of data comparing the effects of warning stimulus properties on saccade latencyfor example, it has not been demonstrated that a warning effect can be obtained with stimulus onsetExperiment 1 examined the relative efficacy of stimulus onset, offset, and change as warning events. Four warning stimulus conditions were compared at warning intervals of $0,100,300$, and 600 msec. For three groups, a blank fixation area was defined by four corner diagonal lines, with warning signal onset and offset consisting, respectively, of the onset or offset of a letter $O$ within the fixation lines. For the third group, the warning interval was signaled by the change of the letter $O$ to a \# symbol for half of the subjects and the reverse change for the other half. Thus, this group always had a symbol in the center fixation area with the warning signal event a stimulus change. Diagonal fixation lines were always present for these three groups in order to avoid confounding warning signal onset and offset with the presence and absence of a fixation reference in the subject's visual field. To provide a condition comparable to the usual visual warning event, fixation stimulus offset, a fourth group was included that had the offset of the letter $\mathrm{O}$ as a warning signal but no diagonal fixation lines present. Two no-warning comparison groups were also tested under conditions where there were no changes in the fixation stimuli and, thus, no warning event preceding the occurrence of the target.

\section{EXPERIMENT 1}

\section{Method}

Subjects. The subjects were 48 undergraduate students ( 24 males and 24 females) enrolled in the introductory psychology course at the University of Wisconsin. All had 20/20 uncorrected near vision in both eyes, as measured by a Titmus Vision Tester, and all received extra course credit for their participation in the study.

Apparatus. The experiment was conducted in a two-room laboratory suite. The subject room was divided by a black curtain extending from floor to ceiling. A portion of the display screen, a Hewlett-Packard Model 1321A display oscilloscope with a fast (P15) phosphor, was visible through a $2.5 \times 36.5 \mathrm{~cm}$ slot in a black panel located in the plane of the curtain. All stimuli presented on the screen had an intensity of 1 nit. The subject was seated in an ophthalmological examination chair equipped with an adjustable headrest. The chair was positioned so that the slot in the cardboard panel was at the subject's eye level at a viewing distance of $60 \mathrm{~cm}$. During the experiment, the subject room was dark except for a single $15-\mathrm{W}$ incandescent bulb located behind the curtain.

The stimulus-generating and response-recording equipment was located in an adjacent control room. An intercom system permitted communication between the experimenter in the control room and the subject. The stimuli presented on the display screen were generated and timed by a laboratory computer.

The subject's eye movements were detected using a Narco Biometric Eye Trac Model 200 eye-movement monitor. Horizontal eye movements were recorded from the right eye, and vertical eye movements were recorded from the left eye. Permanent records of the unfiltered analog output of the Eye Trac were made using a Beckman R511A dynograph. During each trial, the unfiltered Eye Trac output for the right eye (horizontal eye movements) was digitized by the laboratory computer, which identified the onset of the first saccade and determined its latency. At the conclusion of the trial, the trial conditions and saccade latency were printed, and the saccade, with the computer-determined saccade onset identified, was displayed on a monitor scope for the experimenter's inspection.

Design. All subjects received 96 trials. On half of the trials, the target letter $\mathrm{X}$, which subtended visual angles of .5 deg vertically and $.35 \mathrm{deg}$ horizontally, appeared $15 \mathrm{deg}$ to the left of the center of the screen, and on the other half of the trials appeared $15 \mathrm{deg}$ to the right. The target duration was $1 \mathrm{sec}$, and the occurrence of left and right targets was arranged according to Fellows (1967) sequences.

The subjects were divided into six groups, each composed of four males and four females. For four of these groups, the 96 trials included 24 with each of four warning intervals, $0,100,300$, and $600 \mathrm{msec}$. The sequence of warning intervals was random, with the restrictions that within each block of 32 trials each warning interval occur four times with a left target and four times with a right target and that the same type of trial, considering both warning interval and target direction, not occur twice in succession. For the 0 -msec-warning-interval condition, the warning event occurred simultaneously with the onset of the target, while the warning event occurred 100,300 , or $600 \mathrm{msec}$ prior to target onset for the other three warning intervals. In the case of three of these groups-Onset, Offset, and Change-a fixation pattern was present on the screen continuously throughout the trials. This fixation pattern consisted of four diagonal lines, each subtending $.43 \mathrm{deg}$ of visual angle. These lines radiated outward, at angles of $45 \mathrm{deg}$ to the horizontal, from the four corners of an imaginary square subtending $1.32 \mathrm{deg}$ of visual angle on each side. The resulting pattern resembled an $\mathrm{X}$ with the center blanked out. For the Onset group, the appearance of the letter $O$ served as the warning signal. This letter, which subtended visual angles of $.5 \mathrm{deg}$ vertically and .35 deg horizontally, appeared within the blank area at the center of the fixation lines and disappeared with target offset. For the Offset group, the fixation pattern consisted of the diagonal lines plus the letter $O$ within the lines, with the offset of the letter serving as the warning signal. The $O$ reappeared within the fixation lines at the time of target of fset. For the Change group, the fixation pattern consisted of the lines plus the character $\mathbf{O}$ for half of the group, and the lines plus the character \# for the other half. At the beginning of the warning interval, this center character changed, from $\mathrm{O}$ to \# or from \# to $\mathrm{O}$, returning to the original fixation character at target of fset.

For the fourth warning group, No-Lines Offset, the fixation 
pattern consisted only of the letter $O$ at the center of the screen. The offset of this character served as the warning signal. The $O$ reappeared at the center of the screen at the time of target of set. Thus, the stimulus conditions for the No-Lines Offset group and the Offset group were the same except for the presence or absence of the diagonal line fixation pattern.

The two No-Warning groups saw the same stimuli as the Onset and Offset groups described above. However, for these two NoWarning groups there was no warning event since the fixation pattern, either the lines alone or the lines with a letter $O$ at the center, remained on the screen continuously throughout the session.

Procedure. Upon arriving at the laboratory, all subjects received a vision test and were then seated in front of the display screen and told that from time to time the letter $\mathrm{X}$ would appear somewhere in the narrow window. The fixation stimulus was pointed out, and the subject was instructed to look at the $X$ as quickly as possible each time that it appeared. The subjects were asked to sit still, and were encouraged to blink their eyes normally. The sensors were then fitted to the subject and adjusted as necessary, and the lights in the subject room, except for the 15-W lamp, were turned off. After a 2-min period, the eye-movement monitoring equipment was calibrated by having the subject look at various numbers in a horizontal five-number array. This procedure took about $5 \mathrm{~min}$, and trials began approximately $2 \mathrm{~min}$ after the disappearance of the calibration array.

Immediately prior to each trial, the subject's eye position was checked by the computer. If the subject's gaze was not within 1 deg of the center of the fixation area, or if the subject's eyes were closed, the trial was aborted and attempted again approximately $3 \mathrm{sec}$ later. If the subject's gaze still was not within the fixation area, trials were halted briefly and, if necessary, the Eye Trac sensors were recentered to compensate for changes in the subject's head position. Following each trial, the experimenter examined the tracing on the monitor ossciloscope and called for the next trial. This was followed by a computer-controlled pause of variable duration, causing the trials to occur at intervals at 6,10 , or $14 \mathrm{sec}$, with an average intertrial interval of $10 \mathrm{sec}$.

\section{Results}

Each subject's record was examined to determine which trials, if any, should be deleted. Trials were deleted if a blink occurred between the onset of the warning signal and the target, if the first saccade began during the warning interval, if the first saccade was in the direction opposite to the location of the target, or if the first saccade was followed by a corrective saccade of more than $5 \mathrm{deg}$. In addition, trials were not used if the latency of the first saccade was less than $130 \mathrm{msec}$ or greater than $500 \mathrm{msec}$.

For the four warning-interval groups, each subject's average response latency at each of the four warning intervals was determined separately for left and right targets. Since there were no systematic differences between left and right latencies, the two were averaged for each subject. Group averages of these latencies are shown in Figure 1.

For the two No-Warning groups, each subject's average latency was computed in the same manner as those of the warning-interval groups. Since there were no systematic differences in latencies for the two target directions, the two averages were combined. The mean latencies of these two groups were virtually identical, $289.9 \mathrm{msec}(\mathrm{SD}=22.4)$ for the lines-alone group and $286.6 \mathrm{msec}(\mathrm{SD}=31.4)$ for the group shown the lines with the letter in the center of the fixation area. The combined average for these two groups, representing the average reaction time in the absence of any warning signal stimulus change, is indicated by the horizontal line in Figure 1.

The mean latencies for the four groups were analyzed by means of a three-factor analysis of variance, with sex of subject and experimental group as betweensubjects factors and the four warning intervals as a within-subjects factor. There were significant main effects of groups $[F(3,24)=9.58, p<.025]$ and warning interval $[F(3,72)=113.16, p<.001]$ and a significant interaction between these two factors $[F(9,72)=4.47, p<.05]$.

Subsequent planned $t$ tests compared the four groups at each warning interval. There was no significant difference between the onset and change groups at any of the four intervals $[\mathrm{t}(72)=.08,1.54$, .31 , and $.46, \mathrm{p}>.10$ for all four comparisons]. The Offset and No-Lines Offset groups were not significantly different at the 0 -msec warning interval [t(72) $=1.74, \mathrm{p}>.05$ ], but the differences between these two groups were significant at 100 -, 300-, and $600-\mathrm{msec}$ warning $[\mathrm{t}(72)=2.42,2.75$, and 3.56 , respectively, $p<.02$ for all three comparisons]. The differences between the Offset group and the Onset group at the 0 - and 100-msec-warning intervals were significant $[\mathrm{t}(72)=6.43$ and 4.81 , respectively, $\mathrm{p}<.001$ for both]. At the 300- and 600-msec-warning intervals, the Offset group is not significantly different from the Onset group $[t(72)=1.91$ and .27 , respectively, $p>.05$ for both].

Additional $t$ tests examining the warning-interval

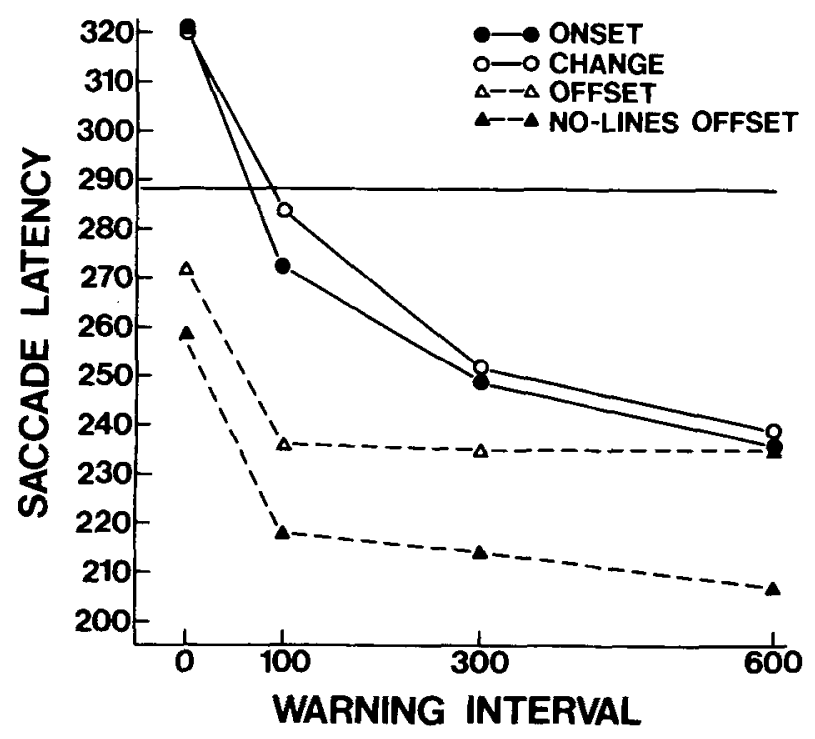

Figure 1. Mean saccade latency as a function of warning interval and warning event type. The horizontal line is the mean latency of no-warning control groups. 
effects within each group show a significant decrease in latency from the 0 -msec warning to the $100-\mathrm{msec}$ warning and from the $100-\mathrm{msec}$ warning to the 300 -msec warning in both the Onset group [t(72) = $6.37, p<.001$, and $t(72)=3.05, p<.01]$ and the Change group $[\mathrm{t}(72)=4.91$ and $4.28, \mathrm{p}<.001$ for both]. In the Offset and No-Lines Offset groups, there was a significant decrease in latency between the 0 -msec warning and the $100-\mathrm{msec}$ warning $[\mathrm{t}(72)$ $=4.72$ and $5.42, \mathrm{p}<.001$ for both] and no further significant decrease as warning interval increased.

Response latencies under the various warning conditions were compared to the average latency of the No-Warning subjects by means of a priori $t$ tests. Since the Onset and Change groups did not differ at any warning interval, they were combined for these comparisons. The average latency of the combined Onset and Change groups was significantly greater than that of the No-Warning latency at 0 -msec warning $[\mathrm{t}(30)=2.88, \mathrm{p}<.01]$, not different from the No-Warning level at $100-\mathrm{msec}$ warning $[\mathrm{t}(30)=.91$, p > .20], and significantly shorter than the NoWarning latency at the 300 - and 600 -msec-warning intervals $[\mathrm{t}(30)=3.46$ and 5.14 , ps $<.01$ and .001 , respectively]. Since the Offset and No-Lines Offset groups were not significantly different at the $0-\mathrm{msec}$ warning, these two groups were combined and compared to the No-Warning subjects. The latency for the combined Offset groups was significantly shorter than the No-Warning latency $[\mathrm{t}(30)=2.37, \mathrm{p}<.05]$. At the remaining warning intervals, where the two Offset groups did differ, each was compared to the No-Warning level separately, and in each case, the latency under warning conditions was significantly shorter than under no-warning conditions [ $t(22)=$ $4.50,5.08$, and 4.44 for comparisons involving the Offset group, and $t(22)=5.73,5.92$, and 7.95 for comparisons involving the No-Lines Offset group, $\mathrm{p}<.001$ for all six tests] .

\section{Discussion}

These data demonstrate the facilitating effect of warning events on the latency of saccadic responses to a peripheral target. Furthermore, stimulus offset was found to be superior to stimulus onset or change, since the Offset and No-Lines Offset groups showed significantly faster responding than the Onset or Change warning groups at a number of warning intervals.

It is also clear that either stimulus onset or change, as well as stimulus offset, is a sufficient warning event for a reduction in saccade latency. This is demonstrated by the fact that both the Onset and Change groups' saccades had significantly shorter latencies than the combined No-Warning group at both 300- and 600msec-warning intervals. These results lead to the conclusion that the postulated reduction in microsaccades following fixation stimulus offset, with the consequent decrease in the refractory effects of such microsaccades on the programming or execution of the subsequent target saccade, is not a necessary condition for the effects of a warning signal on saccade latency. The data do not rule out the possibility, however, that a reduction in microsaccades following the offset of a fixation stimulus does affect saccade latency under some conditions. In this respect, it should be noted that the NoLines Offset group, which had no stimuli remaining on the display after warning signal offset, had significantly shorter latencies than the Offset group, which had the fixation lines remaining in foveal view. In any case, however, such a factor could not account for the warning effect obtained under Onset and Change warning stimulus conditions or, therefore, the larger part of the facilitatory effect obtained with warning stimulus offset. Thus, reduced saccade latency following warning might best be considered to reflect a general preparatory or alerting process that affects the processing of target information, or possibly the preparatory steps related to the programming of the saccade to the target stimulus that follows.

There are indications in the data that a 0 warning interval, that is, the simultaneous presentation of a warning event and the target, is not the equivalent of a no-warning condition, in that the latency of the combined Offset and No-Lines Offset groups was significantly less than that in the No-Warning control condition. Saslow (1967) reported a similar finding in that saccade latency under synchronous fixation light offset and target onset was less than was the case when fixation offset overlapped target onset by several hundred milliseconds.

This reduced saccade latency under simultaneous warning signal and target presentation could reflect the effects of a reduction in microsaccades following warning signal offset, as discussed above, or it could also be due to differences in the transmission of onset and offset information. Since the target was a stimulus onset and the warning signal a stimulus offset, their occurrence may not have been simultaneous to the subject, at some level of stimulus processing, if offsets are indeed perceived earlier than onsets as indicated by studies of temporal order discrimination. It is also possible that even with simultaneous registration of warning offset and target onset, the warning offset might have a facilitatory effect on the programming of the saccade for some period of time after the target initiates such programming.

A possibly more interesting finding is the longer latency of the Onset and Change groups as compared to the No-Warning group under 0-interval-warning conditions. This increase in latency relative to the NoWarning control value could not be attributed to a perceived onset-offset difference and a corresponding difference in the initiation of warning or alerting processes. If the onset or change event simply lagged the offset warning event, shorter latencies would be 
expected to stimulus offset, as was found, but the response to onset or change would not be expected to be slower than that of the No-Warning control condition. Thus, an active inhibitory or interfering effect that overrides the warning effect under 0-warninginterval conditions is indicated in the case of warningstimulus onset.

In order to determine the replicability of the onset and offset differences, as well as to investigate their effects relative to a no-warning condition at values around synchrony of warning and target signals, two additional groups of eight subjects each were tested under the onset and offset conditions of Experiment 1 , but with warning intervals of $-150,-100$, $-50,0,50,100$, and $150 \mathrm{msec}$, where negative values refer to the presentation of the target before the warning event. As in Experiment 1, the Offset group had significantly $(\mathrm{p}<.05)$ shorter saccade latencies than the Onset group. Furthermore, the Onset group was slower than the No-Warning group of Experiment 1 at $0-,-50-,-100-$, and -150 -msec intervals, and significantly so $(\mathrm{p}<.05)$ at the $-50,-100$, and -150 values. In contrast, the Offset group did not differ from the No-Warning value except at positive intervals where responding was faster, again as in Experiment 1.

These data thus support the finding of an interfering effect on saccade latency of a stimulus onset occurring simultaneously with or, in the case of these additional data, up to $150 \mathrm{msec}$ after the onset of a peripheral target. However, at the greatest overlap $(-150 \mathrm{msec})$, the Onset and Offset groups were still significantly different, and the difference between Onset and No-Warning was still present, indicating that even greater overlap would be necessary before the point would be reached at which differences from the No-Warning value would no longer occur.

\section{EXPERIMENT 2}

Experiment 2 was designed to compare the effects of onset and of fset warning stimuli, relative to NoWarning, at target and warning stimulus overlap intervals equal to and greater than those previously employed. Accordingly, Experiment 2 was carried out with eight warning intervals ranging from -300 to $50 \mathrm{msec}$, in 50 -msec steps, with a within-subjects no-change control condition included to provide a no-warning latency value.

\section{Method}

Subjects. The subjects were 16 undergraduate students $(8$ males and 8 females) enrolled in the introductory psychology course at the University of Wisconsin. All had 20/20 uncorrected near vision in both eyes, as measured by a Titmus Vision Tester, and all received extra course credit for their participation in the study.

Apparatus. This study was conducted using the same apparatus employed in Experiment 1.

Design. The subjects were divided into two groups of four males and four females each. One group was run under the Onset and

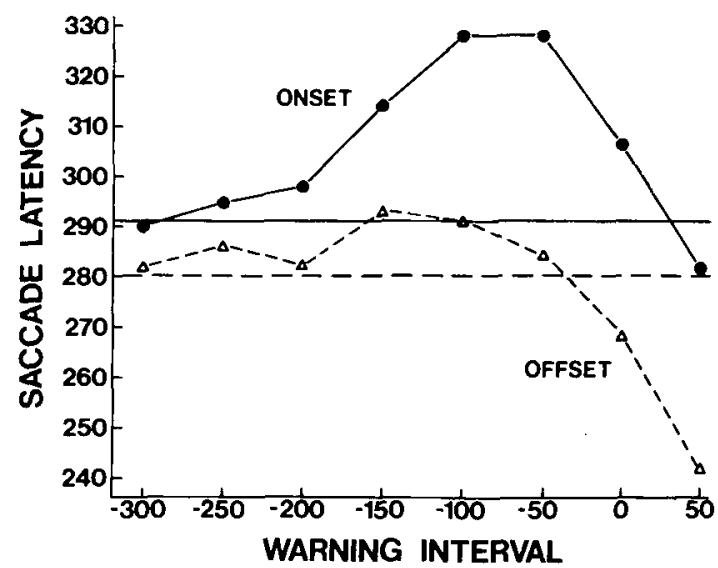

Figure 2. Mean saccade latency of the onset and offset warning groups as a function of warning interval, with negative intervals indicating target onset preceding the warning event. The horizontal lines indicate the saccade latency of each group on trials when no warning event occurred.

the other under the Offset stimulus conditions from Experiment 1. All subjects received 144 trials. The target appeared $15 \mathrm{deg}$ to the left of the center of the screen on half of the trials and 15 deg to the right of center on the other half of the trials, with the occurrence of left and right targets arranged according to Fellows (1967) sequences. The 144 trials included 16 trials under each of 9 warning conditions. The 9 warning conditions included 6 negative warning intervals, $-300,-250,-200,-150,-100$, and $-50 \mathrm{msec}$, in which the warning event occurred after the onset of the target, a 0 -msec warning interval in which the warning event occurred simultaneously with target onset, a 50-msec warning interval, and a no-warning condition in which no warning event occurred. In this last condition, the fixation pattern remained unchanged as the target appeared and disappeared. These 9 warning interval conditions were randomly arranged within blocks of 36 trials with the restrictions that within each block each warning condition occur twice with a left target and twice with a right target and that the same type of trial not occur twice in succession.

Procedure. The procedure was the same as that of Experiment 1.

\section{Results}

Subjects' records were examined and trials deleted according to the same criteria used in Experiment 1. Each subject's average latency under each warning condition was determined separately for left and right targets. Since there were no systematic left-right differences, the two averages were combined. Average latencies for the two groups are shown in Figure 2. The solid and dashed horizontal lines represent the average no-warning latency for the Onset group and Offset group, respectively.

Average saccade latencies were analyzed by means of an analysis of variance with stimulus onset vs. offset and sex of subject as between-subjects factors, and warning intervals as a within-subjects factor. The analysis resulted in a significant main effect of warning interval $[F(8,96)=8.44, p<.01]$ and a significant interaction between onset vs. offset and warning $[F(8,96)=2.30, p<.05]$. Subsequent planned $t$ tests indicated no significant difference between Onset and Offset groups under no warning 
conditions $[\mathrm{t}(96)=1.12, \mathrm{p}>.20]$ or with warning intervals of $-300,-250$, and $-200 \mathrm{msec}[\mathrm{t}(96)=$ $.75, .90$, and 1.54 , respectively, all ps $>.10$ ]. The two groups were significantly different at the remaining warning intervals $[\mathrm{t}(96)=2.15,3.81,4.44,3.90$, and 4.03 at warning intervals of $-150,-100,-50,0$, and 50, respectively, all ps $<.05$ ]. Comparisons of each group to its own no-warning latency indicated that the Onset group's latencies at warning intervals of $-150,-100$, and -50 were significantly greater than the no-warning latency $[\mathrm{t}(96)=2.33,3.76$, and 3.70 , respectively, all ps $<.02]$. At all other warning intervals, the Onset group's average latency was not significantly different from its no-warning level. The Offset group differed from its no-warning level only when the warning interval was $50 \mathrm{msec}[\mathrm{t}(96)=2.06$, $\mathrm{p}<.05]$.

\section{GENERAL DISCUSSION}

The data of Experiment 2 replicate and extend the findings of Experiment 1. The warning signal onsetoffset difference was again obtained, in Experiment 2 for warning interval values from -150 to $50 \mathrm{msec}$, while the finding of an increased latency of response following warning signal onset was also replicated, the comparison in Experiment 2 involving a withinsubjects no-warning condition and the demonstration of significant differences at warning intervals of $-50,-100$, and $-150 \mathrm{msec}$.

The onset-offset latency differences were also comparable across Experiments 1 and 2, the values being 48 and $36 \mathrm{msec}$ for 0 - and 100-msec-warning intervals in Experiment 1, and 38 and $40 \mathrm{msec}$ for 0 - and 50 msec-warning intervals in Experiment 2. While the Experiment 2 Offset group's saccade lattency was slightly greater at some negative warning intervals than it was with no warning, none of the differences were significant. In contrast, the Onset group showed significantly longer latencies with negative warning intervals as compared to its no-warning value.

It is of some interest that the onset-offset difference was found for negative warning intervals up to a 150 msec overlap, this being well beyond the point at which warning-signal offset resulted in a reduction in saccade latency. The fact that the difference at these intervals was due to the Onset group's slower responding relative to their no-warning latency suggests that the major portion of the onset-offset difference was due to an interfering factor associated with warning signal onset rather than a facilitatory factor associated with warning stimulus offset.

From an adaptive point of view, it is not surprising that a saccade should be delayed when new information has been presented at the eye's fixation point. Relevant to this is the suggestion of Potter and Levy (1969), Potter (1976), and Senders (1976) that the eye remains relatively fixed at the place information is rapidly being presented in order to process the changing stimulus information. It is impossible to determine from the present data whether the longer delay following stimulus onset reflects such a process, whose function is related to the encoding of new information and its buffering to protect it from supression effects of subsequent fixations, or simply is the result of incompatability between processes initiated by stimulus onset or change and the programming or execution of the following saccade. An independent question is the locus of the delay mechanism. One possibility would be a more or less automatic process that delays eye movements for an interval following the occurrence of new information in the foveal field. Alternatively, a new stimulus could elicit a microsaccade that might or might not have some utility with respect to fixating the stimulus. A small saccade to the new stimulus, or for that matter a more or less random microsaccade, could occur well before the peripheral target saccade and thus interfere with its programming or execution. It is also possible that a warning signal onset or change event could initiate processing sequences that interrupt or delay ongoing saccade programming without small saccades being involved. In any case, it should be noted that the response latencies of the Onset and Offset groups converge at the longer warning intervals where such factors would not overlap the programming or execution of the saccade to the peripheral target.

The possibility that such mechanisms are involved in the warning stimulus onset effects found in the present studies is highly speculative, and evaluation is not possible from the present data. Obviously, the actual measurement of small saccades under the stimulus condition of this study would provide evidence to evaluate their possible role in the obtained effects.

It also would be of interest to determine if the same onset-offset warning effects could be obtained with motor responses other than saccades. To obtain preliminary data relevant to this question, two groups of subjects were tested under the Onset and Offset stimulus conditions of Experiment 1, but with the required response the manual movement of $13.75-\mathrm{cm}$ vertical handle through a $20-\mathrm{deg}$ arc to the right, or to the left, upon the right or left occurrence of the target stimulus. With this manual response, there was a significant warning-interval effect, but no significant onset-offset difference was found and, in fact, response latency with warning onset was somewhat less than with warning offset. Thus, the onset-offset effects found in the present studies may be specific to eye movements. This would not be particularly surprising given the particular function of saccades in the efficient extraction of information from stimuli that appear in the visual field, as compared to the 
manual response's effector function on the environment.

It should again be pointed out that the onset-offset differences were superimposed upon general warning effects, that is, significant warning in terms of decreased saccadic latencies was found with stimulus onset and change, as well as with stimulus offset. Presumedly, these general warning effects are similar to the facilitatory alerting or preparation processes described by Nickerson (1973) and Posner (e.g., 1975), but it is unclear if the particular alerting mechanisms that have been postulated to underlie the warning effect in other reaction-time situations also apply to the saccadic response system, given its particular functions and characteristics.

In summary, these experiments (1) demonstrate that stimulus onset and change as well as stimulus offset can serve as warning events in reducing the latency of saccades to peripheral targets, (2) clearly show that the facilitating effects of warning are less with onset and change as compared to the offset of warning events, (3) provide an indication that the offset of foveal fixation stimuli may increase the warning effect somewhat, and (4) strongly suggest the existence of an interfering effect consequent to warning stimulus onset or change that increases saccade latency when the warning event occurs simultaneously with, or up to $150 \mathrm{msec}$ after, peripheral target onset.

\section{REFERENCES}

Becker, W. The control of eye movements in the saccadic system. In J. Dichgans \& E. Bizzi (Eds.), Cerebral control of eye movements and motion perception. Basel, Switzerland: Karger, 1972.
Carpenter, R. H. S. Movements of the eyes. London: Peon, 1977.

Cohen, M. E., \& Ross, L. E. Saccade latency in children and adults: Effects of warning interval and target eccentricity. Journal of Experimental Child Psychology, 1977, 23, 539-549.

Cohen, M. E., \& Ross, L. E. Latency and accuracy characteristics of saccades and corrective saccades in children and adults. Journal of Experimental Child Psychology, 1978, 26, 517-527.

Fellows, B. J. Chance stimulus sequences for discrimination tasks. Psychological Bulletin, 1967, 67, 87-92.

Hansteen, R. W. Visual latency as a function of stimulus onset, offset, and background luminance. Journal of the Optical Society of America, 1971, 61, 1190-1195.

Lewis, J. H., Dunlap, W. P., \& Matteson, H. H. Perceptual latency as a function of stimulus onset and of fset and retinal location. Vision Research, 1972, 12, 1725-1731.

NiCKERSON, R. S. Intersensory facilitation of reaction time: Energy summation or preparation enhancement? Psychological Review, 1973, 80, 489-509.

Posner, M. I. Psychobiology of attention. In M. Gazzaniga \& C. Blakemore (Eds.), Handbook of psychobiology. New York: Academic Press, 1975.

PotTer, M. C. Short-term conceptual memory for pictures. Journal of Experimental Psychology: Human Learning and Memory, 1976, 2, 509-522.

Potter, M. C., \& Levy, E. I. Recognition memory for a rapid sequence of pictures. Journal of Experimental Psychology, $1969,81,10-15$.

Sanders, A. F. Foreperiod duration and the time course of preparation. Acta Psychologica, 1972, 36, 60-71.

Saslow, M. G. Effects of components of displacement-step stimuli upon latency for saccadic eye movement. Journal of the Optical Society of America, 1967, 57, 1024-1029.

Senders, J. W. Speculations and notions. In R. A. Monty \& J. W. Senchas (Eds.), Eye movements and psychological processes. Hillsdale, N.J: Erlbaum, 1976.

WALSH, T. Visual onset and of fset latencies. Quarterly Journal of Experimental Psychology, 1973, 25, 154-162.

(Received for publication July 5, 1979; revision accepted October $5,1979$. 